\title{
ASSOCIAÇÃO ENTRE MATURAÇÃO E DESEMPENHO DO SALTO VERTICAL EM JOVENS VOLEIBOLISTAS
}

Jefferson Eduardo Hespanhol

Miguel de Arruda

Joel Moreira Prates

Fabio Henrique Mathias

\section{Resumo}

O objetivo desses foi estimar a contribuição da composição corporal e do status da maturidade na variação no desempenho dos saltos verticais de jovens voleibolistas. Os participantes foram 43 jogadores voleibolistas Brasileiros de idade entre 13 a 19 anos. Os desempenhos dos saltos verticais foram verificados com as técnicas: squat jump, countermoviment e continuun jump 5 seconds. A análise de regressão linear simples foi usada para estimar a contribuição do estágio de maturidade sexual na variação do desempenho. Estágio de puberdade, massa corporal, área muscular da coxa contribuem significantemente, mas em diferentes contribuições nas variâncias no desempenho do salto vertical para todos os testes, todavia, expressando uma pequena 5 a $14 \%$ variação no desempenho $(p<0,05)$.

\section{Palavras-Chaves}

Maturação; Saltos verticais; Voleibol.

\section{MATURITY-ASSOCIATED VARIATION IN VERTICAL JUMP PERFORMANCE OF YOUTH VOLLEYBALL PLAYERS}

Jefferson Eduardo Hespanhol

Miguel de Arruda

Joel Moreira Prates

Fabio Henrique Mathias

\begin{abstract}
The aim of this study was estimate the contribution of body composition and maturity status to variation in jump performance of adolescent volleyball players. The participants were 43 Brazilian volleyball players between 13 to 19 years old. The vertical jump performances were verified with the techniques: squat jump, countermoviment e continuun jump 5 seconds. The analysis of simple linear regression was used esteem the contribution of the period of training of sexual maturity in the variation of the performance. Stage of puberty, body mass, thigh muscular area contributed significantly but in different contribution to variance in vertical jump performance for all the tests, however, expressing a little 5 to $14 \%$ variation $(\mathrm{p}<0,05)$. Conclusively, body composition, and stage of puberty contributed relatively little to variation in performance of adolescent volleyball players.
\end{abstract}

\section{Key-Words}

Maturation; Jump vertical performance; Volleyball. 


\section{INTRODUÇÃO}

Diante das mudanças no desempenho físico, técnico e tático que ocorrem ao longo do tempo na formação dos jovens atletas voleibolistas, tanto de ordem estrutural quanto de ordem funcional (BENETTI; SCHNEIDER; MEYER, 2005; DUNCAN; WOODFIELD; AL-NAKEEB, 2006; MALINA et al., 2005), e da necessidade do desenvolvimento para o alto nível da modalidade, o voleibol tem passado cada vez mais pelo estudo e sistematização de elementos relativos a duas realidades interdependentes: o jogo e o jogador.

No que se referem ao jogo, vários elementos diferenciam o sucesso das equipes, sendo um deles o desempenho físico, cujo principal componente é a altura saltada nas situações de ataque, bloqueio, saque com salto, levantamento com salto (HERTOGH; HUE, 2002; SCATES; LINN, 2003; MALATESTA. et al., 2003; TILLMAN. et al., 2004; KATIC;GRGANTOV; JURKO, 2006) Quanto ao jogador, a importância está relacionada principalmente ao desenvolvimento das capacidades condicionantes associadas a maturação e crescimento, que permitem aumentos dos saltos verticais, assim como, aumentos da velocidade nas ações de deslocamento (MALATESTA et al., 2003).

As alterações significativas no rendimento desempenho do salto vertical são caracterizadas pelas mudanças na função neuro-muscular, tais como: força máxima, força explosiva, força explosiva elástica e reflexa (NEWTON; KRAEMER; HÄKKINEN, 1999). Em vários estudos foram encontrados fortes relacionamentos do desempenho do salto vertical com essas capacidades condicionantes de força (YOUNG; WILSON; BYRNE, 1999; CICCARONE; MARTELLI; FONTANI, 2000). Logo, as manifestações da força vêm cada vez mais se destacando como uma variável importante no desempenho físico dos atletas de voleibol.

Um fator preocupante aos estudiosos está na busca do treinamento compatível de força ao desenvolvimento do jovem atleta, que tendem a desenvolver informações sobre programa de treinamentos efetivos para a capacidade condicionante da força (BLIMKIE, 1992; OZMUN; MIKESKY SURBURG, 1994; FAIGENBAUM; WESTCOTT; MICHELI, 1996; FAIGENBAUM. et al., 1999). Nesse contexto, o desenvolvimento da força explosiva sofre várias mudanças quanto à idade e maturação (VIITASALO, 1982; MALINA; BOUCHARD; BAROR, 2004). Evidencias relatam a existência de aumentos lineares no desenvolvimento da força até a puberdade (MALINA; BOUCHARD; BAROR, 2004), por conseguinte, quando o jovem entra nessa fase de mudanças no desempenho da força, há a partir daí uma marcada aceleração no seu desenvolvimento (MALINA et al., 2005; BLIMKIE, 1992; OZMUN; MIKESKY SURBURG, 1994)

CONEXÕES: revista da Faculdade de Educação Física da UNICAMP, Campinas, v. 6, n. 3, p. 55-67, set/dez. 2008. 
Estudos com jovens atletas não estão considerando sistematicamente a influencia potencial da variação no crescimento e maturidade biológica sobre o desempenho físico da força em jovens atletas (MANNO; GIMINIANI, 2003; GABETT et al., 2006) a qual visa contribuir com informações aos treinadores sobre o processo de desenvolvimento e maturação. Outros estudos têm sugerido que conhecendo as contribuições da maturação sobre os componentes de produção de força, estes poderão condicionar aumentos das capacidades de produção de força em jovens atletas para que possam responder de forma efetivas ao aumento do desempenho (HÄKKINEN; MERO; KAUHANEN, 1989; DIALLO et al., 2001; FAIGENBAUM; MILIKEN; WESTCOTT, 2003; BERALDO, 2003).

Esse estudo teve como propósito estimar a contribuição do estágio de maturidade na variação do desempenho dos saltos verticais.

\section{MÉTODO E MATERIAL}

\section{Participantes do Estudo}

Participaram deste estudo 43 voleibolistas do sexo masculino nas faixas etárias de 13 a 19 anos, divididos em três grupos: 14 infantis, 17 infanto-juvenis e 12 juvenis, os quais realizavam treinamentos diários e pertencentes às categorias de um Clube de voleibol participante do Campeonato Estadual - 2006, localizado na região metropolitana de Campinas/SP - Brasil. Os responsáveis pelos atletas assinaram o termo de consentimento, sendo informados sobre os riscos e os benefícios do projeto de pesquisa. $\mathrm{O}$ estudo proposto foi aprovado pelo Comitê de Ética da Universidade Estadual de Campinas. Todos os participantes voluntários foram selecionados a partir dos seguintes critérios: a) ter mais de dois anos de treinamento regular na prática do voleibol, b) não apresentar nenhuma limitação ortopédica.

\section{PROCEDIMENTOS DE MEDIDAS E TESTES}

Variáveis Antropométricas: As medidas antropométricas de estatura (EST) e massa corporal (MC) foram utilizadas para caracterização dos sujeitos estudados (LOHMAN; ROCHE; MARTORELL, 1988). As variáveis que caracterizam a composição corporal: massa de gordura (MG), massa corporal magra $(\mathrm{MCM})$ e o percentual de gordura $(\% \mathrm{G})$ foram estimados através dos métodos de dobras cutâneas. O percentual de gordura foi estimado pela equação proposta por Lohman (1986). A área muscular da coxa (AMCX) foi mensurada seguindo o procedimento descrito por Frisancho (1990). 


\section{VARIÁVEL MATURACIONAL}

Os participantes se auto-avaliaram maturacionalmente (TANNER, 1962; MATSUDO; MATSUDO, 1994). Os estágios de maturidade sexual foram pré-púberes (PR), púberes (PU) e pós-púberes (PO). Como referencial de interpretação foi considerado que o PR representa sujeitos classificados no estágio 1 de pilosidade pubiana (P1) e de desenvolvimento dos genitais (G1); sujeitos no estágio 2, 3 e 4 de pilosidade pubiana (P2, P3 e P4) e de desenvolvimento dos genitais (G1, G2, G4) foram classificados como PU, já os sujeitos no estágio 5 foram classificados como PO.

Variáveis do salto vertical: As variáveis utilizadas neste estudo foram: squat jump (SJ), countermoviment jump (CMJ) e salto verticais contínuos de 5 segundos (CJ5s). Os testes de saltos verticais foram: o salto com meio agachamento partindo de uma posição estática de 5 segundos (SJ), o teste de salto vertical com contramovimento sem a contribuição dos membros superiores (CMJ), e o teste de saltos verticais contínuos com duração de 5 segundos sem a contribuição dos joelhos e membros superiores (CJ5s). Os testes de saltos verticais com as técnicas SJ e CMJ foram realizados com base na padronização descrita por Komi e Bosco (1978), e o CJ5s seguiu o procedimento descrito por Dalleau et al. (2004). As medidas com a técnica de salto vertical SJ e CMJ foram consideradas confiáveis pela literatura especializada (ELVIRA. et al., 2001; MARKOVIC et al., 2004). Para esse estudo, o coeficiente de correlação intraclasse foi calculado para cada variável dos testes, demonstrando níveis altos de confiabilidade $(\mathrm{CMJ}=0,98 ; \mathrm{SJ}=0,99 ; \mathrm{CJ} 5 \mathrm{~s}=0,91)$ no teste/reteste com saltos verticais. $\mathrm{O}$ equipamento empregado na realização das medidas de saltos verticais foi o tapete de contato Jump Test (BOSCO, 1980).

\section{COLETA DE DADOS}

A coleta dos dados foi realizada no início da temporada. Foi solicitado aos atletas que não fosse realizado nenhum tipo de atividade extenuante $24 \mathrm{hs}$ antes da coleta. A coleta das informações relativas aos dados das medidas antropométricas e da força explosiva foi realizada no ginásio de esportes do Clube. Os sujeitos executaram aquecimento de 15 minutos através das ações de: alongamentos, corridas, exercício coordenativo e exercício para ativação neuro-muscular direcionadas ao teste de saltos. Cada sujeito realizou três tentativas máximas para cada um das condições dos testes com saltos verticais: SJ, CMJ e CJ5s. O tempo de recuperação foi de 10 segundos entre as tentativas e 60 segundos entre os testes. O melhor das tentativas de cada técnica de salto vertical SJ e CMJ foi escolhido como o escore de medida. 


\section{PROCEDIMENTO DE ANÁLISE - TRATAMENTO ESTATÍSTICO}

Os dados foram tratados pelas técnicas de estatísticas descritivas (média e desvio padrão) e do teste tStudent para amostras independentes para a comparação entre os estágios maturacionais. A ANOVA fator único com medidas repetidas foi utilizada na comparação das diferenças entre as categorias, seguido do post hoc Tukey's teste para a comparação múltipla das médias. As relações entre as variáveis foram estimadas pela analise de correlação de Pearson produto momento. O nível de significância adotado foi de $\mathrm{p}<0,05$. A análise de regressão linear simples foi usada para estimar a contribuição do estágio de maturidade sexual na variação do desempenho no produto da força estimado pela escala alométrica.

A escala alométrica foi utilizada na observação das respostas da produção das manifestações força em adolescentes em diferentes momentos dos estágios de maturidade sexual com relação a composição corporal. Esta relação pode ser expressa pela seguinte equação alométrica: $\mathrm{Y}=\mathrm{a} \cdot \mathrm{x}^{\mathrm{b}}$. Onde $\mathrm{Y}$ é a variável relacionada ao resultado da produção de força; a é manifestação da produção da força na estimativa da altura do salto, e x é a variável que indicam a composição corporal. O expoente constante (b) utilizado foi de 0,69 para SJ; 0,90 para o CMJ e 0,96 para o CJ5 s (MARKOVIC; JARIC, 2005).

\section{RESULTADOS}

Estatística descritiva para composição corporal e desempenho da força são resumidas nas tabelas 1 e 2 , respectivamente. Houve tendência de variações nos tecido muscular indicando um maior volume da musculatura nos PO do que PU. Em ambas as técnicas de saltos verticais, os PO saltaram mais alto quando comparado com os PU. No índice de elástica, os PO apresentaram índices com valores elevados do que os PU. Os meninos PO e PU tiveram resultados similares nos índices de reatividade.

Tabela 1 - Demonstrativo das características antropométricas quanto ao estágio maturacional.

\begin{tabular}{cccccc}
\hline \multirow{2}{*}{ Variáveis } & \multicolumn{2}{c}{ PÚBERES (n=10) } & \multicolumn{2}{c}{ PÓS-PÚBERES $(\mathbf{n}=\mathbf{3 3})$} & \multirow{2}{*}{ p } \\
\cline { 2 - 5 } & MÉDIA & DP & MÉDIA & DP & \\
\hline IDADE (anos) & 16,15 & 1,07 & 17,68 & 1,42 & 0,0014 \\
EST (cm) & 185,56 & 6,25 & 190,85 & 7,13 & 0,0372 \\
MC (kg) & 70,15 & 8,76 & 77,34 & 9,45 & 0,0409 \\
\%G & 16,87 & 5,31 & 15,26 & 3,96 & 0,0039 \\
MCM (KG) & 58,00 & 4,98 & 65,48 & 8,00 & 0,0000 \\
AMCX (CM $\mathbf{C M}^{\mathbf{2}}$ & 196,38 & 29,45 & 215,04 & 37,90 & 0,0108 \\
\hline
\end{tabular}

EST=Estatura; $\mathbf{M C}=$ Massa Corporal; \%G= Percentual de Gordura; $\mathbf{M C M}=$ Massa Corporal Magra; $\mathbf{A M C X}=$ Área Muscular da Coxa.

CONEXÕES: revista da Faculdade de Educação Física da UNICAMP, Campinas, v. 6, n. 3, p. 55-67 , set/dez. 2008. 
No relacionamento entre os indicadores de composição corporal e desempenho do salto vertical nas manifestações das técnicas verificou-se que houve significantes diferenças entre PU com o PO, demonstrando que os PO apresentaram maiores alturas saltadas em todos os testes de saltos do que PU.

Tabela 2- Demonstrativo dos desempenhos dos saltos verticais dos participantes quanto ao estágio maturacional.

\begin{tabular}{cccccc}
\hline \multirow{2}{*}{ Variáveis } & \multicolumn{2}{c}{ PÚBERES (n=10) } & \multicolumn{2}{c}{ PÓS-PÚBERES (n=33) } & p \\
& MÉDIA & DP & MÉDIA & DP & 0,0054 \\
\hline SJ(cm) & 32,32 & 3,97 & 36,96 & 3,66 & 0,0019 \\
CMJ (cm) & 35,45 & 3,99 & 41,74 & 3,96 & 0,0021 \\
CJ5s (cm) & 36,18 & 5,05 & 42,35 & 4,08 & 0,0373 \\
IE (\%) & 9,69 & 3,91 & 13,06 & 4,81 & 0,6756 \\
IR (\%) & 2,84 & 0,60 & 2,94 & 0,46 &
\end{tabular}

$\mathbf{I E}=$ Índice de Elasticidade; $\mathbf{I R}=$ Índice de Reatividade.

As estimativas da relativa contribuição do estágio de maturidade sexual, composição corporal no desempenho dos saltos verticais são resumidas na tabela 3. O estágio de puberdade, massa corporal e área muscular da coxa contribuíram significantemente, mas em diferentes contribuições para a variação do desempenho dos saltos verticais para todos os testes, no entanto, expressam uma pequena variância de 5 a $14 \%(\mathrm{p}<0,05)$. 
Tabela 3 - Demonstrativo do desempenho dos saltos verticais correspondendo à escala alométrica quanto ao estágio de maturidade sexual.

\begin{tabular}{ccccccc}
\hline \multirow{2}{*}{ Variáveis } & \multicolumn{3}{c}{ PÚBERES (n=10) } & \multicolumn{3}{c}{ PÓS-PÚBERES (n=33) } \\
\cline { 2 - 7 } & SJ & CMJ & CJ5 & SJ & CMJ & CJ5 \\
\hline \multirow{2}{*}{ MC } & 616,04 & 1655,11 & 1982,91 & 738,19 & 2059,76 & 2428,97 \\
& $\pm 158,80$ & $\pm 445,43$ & $\pm 558,33$ & $\pm 95,20^{\mathrm{a}}$ & $\pm 295,76^{\mathrm{a}}$ & $\pm 446,29^{\mathrm{a}}$ \\
\multirow{3}{*}{ MCM } & 545,13 & 1408,85 & 1666,60 & 654,31 & 1760,74 & 2059,28 \\
& $\pm 139,96$ & $\pm 374,70$ & $\pm 466,59$ & $\pm 87,52^{\mathrm{a}}$ & $\pm 269,10^{\mathrm{a}}$ & $\pm 422,64^{\mathrm{a}}$ \\
\multirow{2}{*}{ AMCX } & 1206,27 & 3976,45 & 5056,42 & 1433,04 & 4899,47 & 6145,73 \\
& $\pm 301,73$ & $\pm 1030,46$ & $\pm 1393,85$ & $\pm 220,18^{\mathrm{a}}$ & $\pm 890,01^{\mathrm{a}}$ & $\pm 1464,99^{\mathrm{a}}$ \\
\hline
\end{tabular}

$\mathbf{M C}=$ Massa Corporal; $\mathbf{M C M}=$ Massa Corporal Magra; $\mathbf{A M C X}=$ Área Muscular da Coxa; ${ }^{\mathrm{a}}=\mathbf{p}<0,05$ em relação à PU;

Observaram que na técnica de SJ teve maior variância do estatuto maturacional na $\operatorname{AMCX}(10 \%, \mathrm{p}<0,05)$ do que $\mathrm{MC}$ e $\operatorname{MCM}(5 \%, \mathrm{p}<0,05)$; no $\mathrm{CMJ}$ notaram significantes variâncias de 11 a $14 \%(\mathrm{p}<0,05)$, respectivamente para $\mathrm{MC}$, MCM, AMCX, como também no CJ5s foram verificadas significantes variância de 5\% para AMCX, 10\%MCM e 12\% MC.

\section{DISCUSSÕES}

Nesse estudo independentemente da categoria e da composição corporal, os voleibolistas de estágio de maturidade PO apresentaram sempre valores superiores aos PU nas várias manifestações da força. Logo, houve aumento da altura saltada em relação ao estatuto maturacional, no entanto, quando comparado com resultados encontrados por adultos (MALINA, 1994), essa relação é fortalecida evidenciando o desenvolvimento da força muscular em jovens voleibolistas em todos os testes de saltos verticais.

Essas diferenças encontradas nos diferentes relacionamentos evidenciam que o estatuto maturacional é um fator que contribui positivamente no nível de desenvolvimento da força muscular (MALINA et al., 2005; MARTIN et al., 2004). A maturação como referência aos fatores qualitativos musculares interfere na forma de manifestação de força, entretanto, o poder de determinação da maturação biológica no desempenho do salto vertical foi pequeno, explicando cerca de 5 a 14\% da variância.

Especificamente a essa variação no SJ a maior contribuição do estatuto maturacional foi com a AMCX, que pôde ser explicada pelos componentes contrateis (FAIGENBAUM; MILIKEN; WESTCOTT, 2003; 
PAASUKE; ERELINE; GPEYEVA, 2000; SEGER; THORSTENSSON, 2000; WOOD et al., 2004; GOROSTIAGA et al., 1999); nota-se, entretanto, que na MC e MCM foi observada uma discreta contribuição (5\%) na variação do desempenho.

O estágio maturacional, MC, MCM e AMCX, contribuíram significantemente na variação do desempenho do CMJ, justificado pela ação do ciclo da musculutara em alongar e encurtar em seu volume muscular (AMCX), assim como, pelas massas corporais influenciando a produção de força explicada por ambos os elementos: elasticidade do tendão e velocidade de ativação dos músculos (MARKOVIC et al., 2004; MARTIN et al., 2004). Também o estágio de maturidade, a MC e MCM contribuíram significativamente na variação do desempenho expressando uma variância pequena na manifestação do CJ5s, apesar disso uma contribuição discreta (5\%) foi notada da $\mathrm{AMCX}$ e os estágios, aqui a rapidez dos movimentos nas fases excêntrico-concêntrica e a transição delas são determinantes nesse tipo de ações musculares (KUBO et al., 2007); ao invés do aumento da AMCX.

Essas diferenças nas manifestações reativas indicam que durante a ação do CMJ, a fibra muscular e a estrutura do tendão executam o trabalho com velocidades mais lentas das fases excêntricas e de transição antes do encurtamento quanto o CJ5s, isto devido à condição da técnica que antecede a execução do movimento. A diferença observada na variação do desempenho pode ser explicada pela velocidade do alongamento, rápida transição entre as fases e a velocidade de contração muscular. O componente reflexo está relacionado às propriedades de velocidade do complexo músculo-tendão (KUBO et al., 2007), as quais são influenciadas pelos níveis de treinamento e habilidade (MALINA et al., 2005).

Considerando esse ponto de pequena variância da maturação biológica na CJ5s, há uma tendência de discreta contribuição da maturação biológica na propriedade do tendão na variação do desempenho do CJ5s em jovens atletas. Consideravelmente, outros fatores deverão ser relevantes nas interações dessas descobertas, especialmente, as mudanças que poderão ocorrer em estudos longitudinais nas manifestações da produção de força em jovens voleibolistas. São necessárias mais pesquisas para explicar esse fenômeno observado, incluindo-se associações como a idade cronológica (MARTIN et al., 2004), os anos de treinamento (MALINA et al., 2005; WOOD et al., 2004; KASABALIS; DOUDA; TOKMAKIDS, 2005), a composição corporal (MALINA et al., 1994; DUNCAN; WOODFIELD; AL-NAKEEB, 2006), relativa estocagem de energia elástica e ação da stiffness no complexo músculo-tendão (DIALLO et al., 2001; MARKOVIC et al., 2004; DALLEAU et al., 2004.

CONEXÕES: revista da Faculdade de Educação Física da UNICAMP, Campinas, v. 6, n. 3, p. 55-67, set/dez. 2008. 
A puberdade é caracterizada pelas mudanças na constituição da $\mathrm{MC}$, logo podemos observar que as diferenças foram significantes entres os estágios maturacionais; isto se refere que os PO tendem aumentar a sua massa em associação ao exercício físico regular, e maturação biológica (MALINA et al., 1994). Estes aumentos de MC para os PO do que PU devem estar relacionados com o aumento da massa muscular (MCM). Houve registro de significantes diferenças entre os grupos na MCM, o que está em concordância com outro estudo (MALINA et al., 2005), cujos estágios de maturidade classificados como PU possuem uma menor MCM quando comparados com os PO.

A composição corporal altera-se consideravelmente durante o processo normal de crescimento, sobretudo na fase pubertária e pós-pubertária (BEILEAU; LOHMAN; SLAUGHTER, 1985). Deste modo, é evidente constatar que outro indicador a AMCX, também demonstrou diferenças na comparação entre PUxPO. O maior volume de AMCX é devido ao volume da musculatura da coxa dos rapazes PO, que está associado ao processo maturacional. Sendo que tal associação é relativamente moderada a forte no período pubertário e forte no pós-pubertário (MALINA et al., 1994; MALINA et al., 2005), os quais somados aos efeitos positivos do exercício físico e o do treinamento no aumento da área muscular dos músculos da coxa resultam em maiores volumes da AMCX do que PU.

\section{CONCLUSÃO}

Os estágios de puberdade contribuem com pequena variação no desempenho das técnicas de saltos verticais em voleibolistas adolescentes. Os voleibolistas em estágio de maturidade sexual classificado como PO apresentaram sempre maiores produções de força do que PU, além disso, demonstraram ter maiores áreas musculares e, conseqüentemente, maiores volumes de massa corporal magra do que os PU. Embora, o engrandecimento do desempenho dos saltos verticais não seja sustentado somente pelo estatuto maturacional, o desenvolvimento da força é possível pelos fatores qualitativos (técnica de execução dos movimentos, níveis de treinamento, condições ambientais), e quantitativos (tamanho corporal, anos de treinamento, idade, composição corporal).

Sugere que no treinamento em jovens atletas diferencie essas técnicas de saltos verticais. Isto pode ser argumentado que houve diferenças na altura do salto vertical entre os estágios de maturidade, e que entre os testes de saltos verticais, houve uma tendência de variações diferentes, pois o SJ tendem a ter uma contribuição maior da AMCX, quanto ao CMJ de MC, MCM, AMCX, e CJ5s de MC, MCM.

CONEXÕES: revista da Faculdade de Educação Física da UNICAMP, Campinas, v. 6, n. 3, p. 55-67, set/dez. 2008. 


\section{REFERÊNCIAS}

BEILEAU, R.; LOHMAN, T.; SLAUGHTER, M. Exercise and body composition of children and youth. Scan J Sports Science, v. 7, n.1, p. 17-27, 1985.

BENETTI, G.; SCHNEIDER, P.; MEYER, F. Os beneficios do esporte e a importância da treinabilidade da força muscular de pré-puberes atletas de voleibol. Revista Brasileira Cineantropometria e Desempenho Humano, v. 7, n. 2, p.87-93, 2005.

BERALDO S. Il miglioramento della forza nell"adolescenza. AtleticaStudi, v. 3/4, p. 65-74, 2003.

BLIMKIE, C. J. R. Resistance during training pre-and early puberty: efficacy, trainability, mechanism, and persistence. Canadian Journal Sport Science, v. 17, p. 264-279, 1992.

BOSCO C. Sei um grande atleta: vediano che cosa dice I'Ergojump. Pallavolo v.5, p.34-36,1980.

CICCARONE, G.; MARTELLI, G.; FONTANI, G. Evaluation of jumping capacities in volley players of different role. Science and Sports, v. 15, p. 332, 2000.

DALLEAU, G. et al. A simple method for field measurements of leg stiffness in hopping. International Journal of Sports Medicine, v. 25, p.170-176, 2004.

DIALLO, O. et al. Effects of plyometric training followed by a reduced training programmer on physical performance in prepubescent soccer players. Journal Sports Medicine and Physical Fitness, v. 41, n. 3, p. 342-48, 2001.

DUNCAN, M. J.; WOODFIELD, L.; AL-NAKEEB, Y. Anthropometric and physiological characteristics of junior elite volleyball players. British Journal Sports Medicine, v. 40, n.7, p. 649-651, 2006.

ELVIRA, J. L. L. et al. Comparative study of the reliability of three jump tests with two measurement systems. Journal of Human Movement Study, v. 41, p. 369-383, 2001.

FAIGENBAUM, A. D.; WeSTCOTT, W. L.; MICHELI, L. J. The effects of Strength training and detraining on children. Journal of Strength and Conditioning Research, v. 10, p. 109-114,1996.

. et al. The effects of different resistance training protocols on muscular strength and endurance development in children. Pediatrics, v. 104, n.1, p. 1-7, 1999.

. et al. Maximal strength testing in healthy children. Journal of Strength and Conditioning Research, v. 17, n.1, p.162-166,2003.

FRISANCHO, A. R. Anthropometric standards for the assessment of growth and nutritional status. Ann Arbor: University Michigan Press, 1990.

GABETT, T. et al. Changes in skill and physical fitness following training in talent-identified volleyball players. Journal of Strength and Conditioning Research, v. 20, n. 1, p. 29-35, 2006. 
GOROSTIAGA, E. M; et al. Effects of heavy resistance training on maximal and explosive force production, endurance and serum hormones in adolescent handball players. European Journal of Applied Physiology, v. 80, p.485-493, 1999.

HÄKKINEN, K.; MERO, A.; KAUHANEN, H. Specificity of endurance, sprint, and strength training on physical performance capacity in young athletes. Journal Sports Medicine and Physical Fitness, v. 29, n.1, p. 27-35, 1989.

HERTOGH, C.; HUE, O. Jump evaluation of elite volleyball players using two methods: jump power equations and force platform. Journal of Sports Medicine and Physical Fitness, v. 42, p. 300-303, 2002. KASABALIS, A.; DOUDA, H.; TOKMAKIDIS, S. P. Relationship between anaerobic power and jumping of selected male volleyball players of different ages. Percept Motor Skills, v. 100, n. 3, p. 607$614,2005$.

KATIC, R.; GRGANTOV, Z.; JURKO, D. Motor structures in female volleyball players aged 14-17 according to technique quality and performance. Coll Antropol, v. 30, n. 1, p. 103-112, 2006.

KOMI, P. V.; BOSCO, C. Utilization of stored elastic energy in leg extensor muscles by men. Medicine and Science in Sports and Exercise, v. 10, n. 2, p. 261-265, 1978.

$\mathrm{KUBO}, \mathrm{K}$. et al. Influences of tendon stiffness, joint stiffness, and electromyography activity on jump performance using single joint. European Journal of Applied Physiology, v. 99, n. 2, p. 235-243, 2007.

LOHMAN, T. G.; ROCHE, A. F.; MARTORELL, R. Anthropometric standardization reference manual. Champaign: Human Kinetics, 1988.

LOHMAN, T. G, Applicability of body composition techniques and constants for children and youth. Exercise and Sports Science Reviews, v. 14, p.325-57, 1986.

MALATESTA, D. et al. Effects of electromyostimulation training and volleyball practice on jumping ability. Journal Strength Conditioning Research, v. 17, n. 3, p. 573-579, 2003.

MALINA, R. M. Physical activity and training: effects on stature and the adolescent growth spurt. Medicine and Science in Sports and Exercise, v. 26, p.759 -766, 1994.

.; BOUCHARD, C.; BAR-OR, O. Growth, maturation, and physical activity. Champaign, Human Kinetics, 2004.

.; et al. Maturity-associated variation in sport-specific skills of youth soccer players aged 13-15 years. Journal of Sports Science, v. 23, n. 5, p. 515-522, 2005.

MANNO, R.; GIMINIANI R. D. Controllo e allenamento della forza muscolare nei bambini e nei giovani. Atletica Studi, v. 3/4, n.1, p. 27-40, 2003. 
MARKOVIC, G. et al. Reliability and factorial validity of squat and countermovement jump tests. Journal of Strength and Conditioning Research, v. 18, n. 3 p. 551-555, 2004.

MARKOVIC, G. et al. Scaling of muscle power to body size: the effect of stretch-shortening cycle. European Journal of Applied Physiology, v. 95, n. 1, p. 11-19, 2005.

MARTIN, R. J. F. et al. Longitudinal changes of maximal short-term peak power in girls and boys during growth. Medicine and Science in Sports and Exercise, v. 36, n. 3, p. 498-503, 2004.

MATSUDO, S.; MATSUDO, V. Self-assessment and physician assessment of sexual maturation in Brazilian boys and girls: concordance and reproducibility. American Journal of Human Biology, v. 6, p. $451-455,1994$.

NEWTON, R. U.; KRAEMER, W. J.; HÄKKINEN, K. Effects of ballistic training on preseason preparation of elite volleyball players. Medicine and Science in Sports and Exercise, v. 31, n. 2, p. 323 $330,1999$.

OZMUN, J. C.; MIKESKY, A. E.; SURBURG, P. R. Neuromuscular adaptations following prepubescent strength training. Medicine and Science in Sports and Exercise, v. 26, p. 510-514, 1994.

PAASUKE, M.; ERELINE, J.; GPEYEVA, H. Twitch contraction properties of plantar flexor muscles in pre and pos-puberal boys and men. European Journal of Applied Physiology, v. 82, p. 459-464, 2000.

SCATES, A. L.; LINN, M. Complete conditioning for volleyball. Champaign: Human Kinetics, 2003.

SEGER, J. Y.; THORSTENSSON, A. Muscle strength and electromyogram in boys and girls followed puberty. European Journal of Applied Physiology, v. 81, n. 1, p.54-61, 2000.

TANNER, J. M. Growth and adolescence. Oxford: Blackwell Scientific Publication, 1962.

TILLMAN, M. D. et al. Jumping and landing techniques in elite women's volleyball. Journal of Sports Science and Medicine, v. 3, p. 30-36, 2004.

YOUNG, W.; WILSON, G.; BYRNE, C. Relationship between strength qualities and performance in standing and run-up vertical jumps. Journal of Sports Medicine and Physical Fitness, v. 39, p. 285-293, 1999.

VIITASALO, J. T. Anthropometric and physical performance characteristics of male volleyball players. Canadian Journal Applied Sports, v. 7, n. 2, p. 182-188, 1982.

WOOD, L. et al. Elbow flexion and extension strength relative to body or muscle size in children. Medicine and Science in Sports and Exercise, v. 36, n. 11, p. 1977-1084, 2004. 


\section{Jefferson Eduardo Hespanhol}

Faculdade de Educação Física/Pontifícia Universidade Católica de Campinas - PUCC

Endereço: Rua Dr. Diogo Prado, 134, Cambuí, ZIP: 13024-210, Campinas, São Paulo, Brasil.

Telefone: (019) 32942984

E-Mail: jeffehespa@hotmail.com

\section{Miguel de Arruda}

Faculdade de Educação Física/UNICAMP

Joel Moreira Prates

Faculdade de Educação Física/UNICAMP

Fabio Henrique Mathias

Faculdade de Educação Física/UNICAMP

Referência do artigo:

ABNT

HESPANHOL, J. E., et al. Associação entre maturação e desempenho do salto vertical em jovens voleibolistas. Conexões, v. 6, n. 3, p. 55-67, 2008.

APA

Hespanhol, J. E., Arruda, M., Prates, J. M., \& Mathias, F. H. (2008). Associação entre maturação e desempenho do salto vertical em jovens voleibolistas. Conexões, 6(3), 55-67.

\section{VANCOUVER}

Hespanhol JE, Arruda M, Prates JM, Mathias FH. Associação entre maturação e desempenho do salto vertical em jovens voleibolistas. Conexões, 2008; 6(3):55-67.

Artigo Recebido 01/10/08

Aceito 17/10/2008 